\title{
EVOLUTION
}

\section{A trip back in time to study cardiorespiratory control}

\author{
Sci Adv 4, eaaq0800 (2018)
}

The connection between heart rate and lung ventilation in mammals is a bit of an evolutionary mystery. A mechanism called respiratory sinus arrhythmia (RSA) causes an increase in heart rate during each intake of breath, and a slowdown during expiration. In young people and very fit individuals RSA is very pronounced.

The strange thing is that these changes in heart rate don't seem to have any obvious physiological function other than a bit more oxygen uptake, and the effect seems too small to justify the complex nervous mechanisms needed to generate it. "People have argued that it may just be that the heart is taking a rest between breaths, but this is really pushing at looking around for functional significance," said Edwin W. Taylor, emeritus professor of animal physiology at the University of Birmingham, UK.

Many physiologists believe this mechanism is unique to mammals, but Taylor is doubtful. Mammalian hearts are undivided, which means they cannot direct more blood flow to the lungs. As a result, blood flow is the same in the lung and in the separate circulatory system, so what would be the point of increasing heart rate in preparation for a breath if it produced no significant increase in blood flow directed to the lungs to receive the incoming oxygen?

The origins of a functional role for RSA may lie with reptiles and amphibians, which do in fact have divided hearts, and they can and do pump more blood to the lungs when they take a breath. That produces more respiratory gas exchange and improved oxygenation of the blood. The lack of any similar relationship in mammals led Taylor to wonder if RSA in mammals may simply be an evolutionary relic of their reptilian ancestry.

A direct test of the idea would require a journey back 400 million or so years ago, to the Devonian era, where an arid landscape and low oxygen levels put pressure on aquatic animals to improve oxygen supply. That resulted in the appearance of the first primitive lung, which allowed its owner to gulp air at the surface of the water.

But a trip to the water surface requires energy and leaves the animal temporarily vulnerable to predators, so these early fish needed to squeeze the most benefit out of each breath. It made sense to Taylor that they would have quickly evolved a system to raise their heart rate with each breath. "Someone with my background would predict that the system we understand very well from the

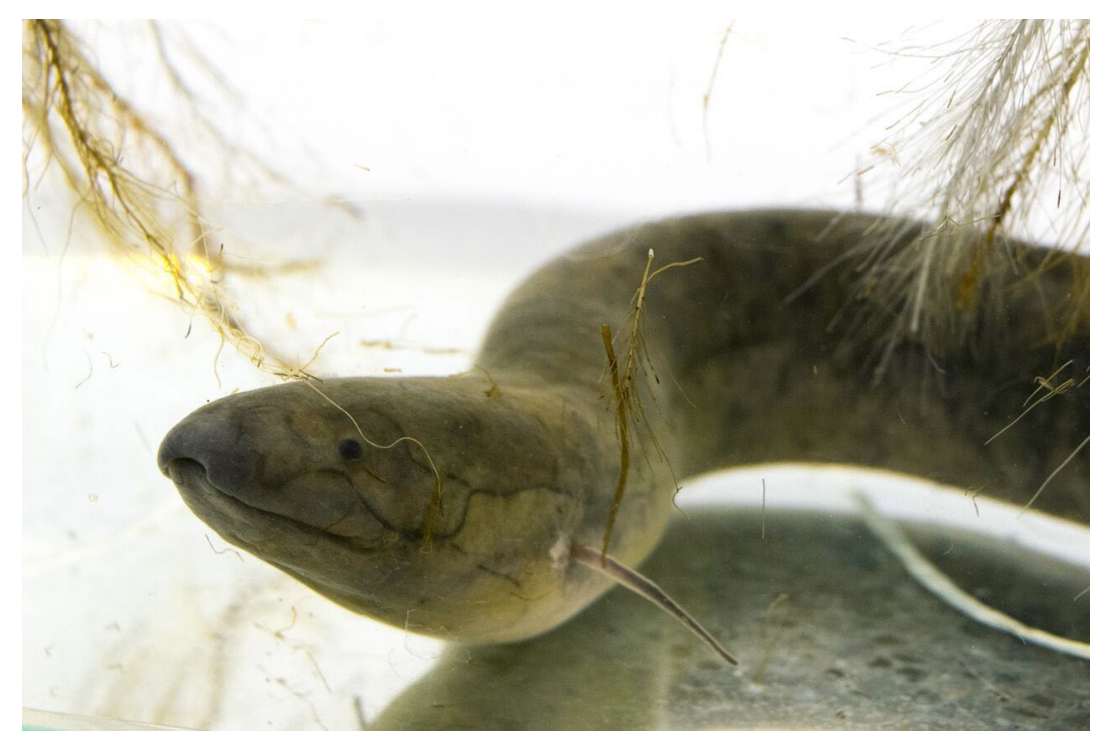

The South American lungfish, Lepidosiren paradoxa. Credit: Gustavo Oda

work done on mammals would show up when the animals began successfully to air breathe," said Taylor. He is at the end of his career, but is passing the baton to a group of young researchers at the Federal University of São Carlos in Brazil, led by Cleo Leite and Diana Monteiro, who collaborated with him on this project and are exploring the physiology of Brazil's fauna.

Lacking a time machine to test their hypothesis with direct observations, the researchers turned to the next best thing: the modern-day South American lungfish, a close relative of that first air-breather. Lungfish reside in shallow, freshwater lakes and rivers that are low in oxygen and may dry up for months at a time. They can survive for up to a year buried in mud, waiting for the next rain. While other relatives of the first air-breathing fish evolved over the past 400 million years into amphibians, reptiles, birds and mammals, lungfishes retained many of the ancient characteristics of the first lunged fish. "They've hung on because they occupy a challenging niche," said Taylor.

The researchers placed lungfish in a tank and inserted electrodes beneath their skin to measure heart rate as they drew in air. Air flow over the surface of the water led to an analyzer, allowing them to measure the oxygen consumption as well. This experiment confirmed that lungfish increase their heart rate at each surface air breath, which leads to a highly significant increase in oxygen uptake. Heart rate control mechanisms are similar to those seen in mammals, including distribution of control areas in the brain. Injection of a nerve block agent showed that the effect is controlled by the vagus nerve, which extends from the brain to the heart. The lungfish nerves conduct signals faster than expected, close to what is seen in mammals, and the speed appears to be due to an insulating myelin sheath that coats the nerve fibers.

The findings could shake up some researchers, who are convinced that the mammalian brain is unique in its control of the heart during respiration, and that such a system is part of what distinguishes the more "advanced" mammalian lineage. "The lungfish is for sure a primitive animal, but mammalian [researchers] are always confusing primitive with simple. They think that reptiles lack sophisticated control systems and that fish simply float around," said Taylor.

He anticipates the conclusion they draw from this study - that RSA and related mechanisms in mammals are likely an evolutionary relic-will get their attention. "This is designed to stir things up," he said.

\section{Jim Kling}

Published online: 23 April 2018

https://doi.org/10.1038/s41684-018-0062-3 